\title{
Cientificismo, analogía y humanidades ${ }^{1}$
}

Salvador Jara Guerrero²

Universidad Michoacana de San Nicolás de Hidalgo

Recibido: 2 de noviembre de 2009 • Aprobado: 5 de marzo de 2010

\section{Resumen}

Se realiza una crítica a las posiciones cientificistas, especialmente en las ciencias sociales y en las humanidades, destacando el valor de la epistemología como herramienta crítica y de las posiciones prudenciales. Se describen los éxitos de la física desde la Revolución científica con el objetivo de contextualizar y valorar adecuadamente el desarrollo de las ciencias naturales, pero sobre todo del método científico, las condiciones en que se aplica de manera general y sus limitaciones, incluso en el estudio de los fenómenos naturales, en los que se reconoce actualmente una gran complejidad, pero nunca igual a la de los fenómenos sociales o a los estudiados en las humanidades.

Palabras clave: cientificismo, epistemología, humanidades, analogía.

1 Este artículo hace parte de las investigaciones en torno a la fundamentación epistemológica de las ciencias sociales.

2 Licenciado en Ciencias Físico Matemáticas de la Universidad Michoacana de San Nicolás de Hidalgo. Cursó la Especialidad en Física Experimental en la Universidad de Berkeley, USA, y la Maestría en Tecnología Educativa en el Instituto Latinoamericano de la Comunicación Educativa. Obtuvo el grado de Maestría en Filosofía de la Cultura en la Universidad Michoacana de San Nicolás de Hidalgo con mención honorífica y el grado de Doctor en Filosofía en la Facultad de Filosofía y Letras de la Universidad Nacional Autónoma de México con mención honorífica. Correo electrónico: sjarag@gmail.com 


\section{Scientism, analogy and humanities}

\section{A bstract}

We present a criticism of scientistic positions, especially in the social sciences and humanities, emphasizing the value of epistemology as a critical tool and prudential positions. It describes the successes of physics in the Scientific Revolution in order to contextualize and properly assess the development of natural sciences, but especially the scientific method, the conditions under which it generally applies and its limitations, even in the study of natural phenomena, in which it is now recognized as very complex, but never equal to that of social phenomena or to study in the humanities.

Key words: scientism, epistemology, humanities, analogy. 


\section{Scientifisme, analogie et humanités}

\section{ésumé}

On réalise une critique des positions scientifistes, en particulier dans les sciences sociales et les humanités, en mettant en évidence la valeur de l'épistémologie comme outil critique et celle des positions prudentielles. On décrit les succès de la physique depuis la Révolution scientifique dans le but de contextualiser et de valoriser de manière adéquate le développement des sciences naturelles, mais avant tout de la méthode scientifique, des conditions dans lesquelles on l'applique de manière générale et de ses limites, y compris dans l'étude des phénomènes naturels, dans lesquels on reconnaît actuellement une grande complexité, mais en aucun cas égale à celle des phénomènes sociaux, ni à ceux qui sont étudiés en sciences humaines.

Mots-clé: scientifisme, épistémologie, humanités, analogie. 


\section{Introducción}

Cacambo, en Cándido de Voltaire, es un personaje que representa la sabiduría particular, práctica y popular que se obtiene con la experiencia de la vida. El otro personaje es el Dr. Panglos, filósofo optimista, pensador teórico, teólogocosmológico, quien sólo habla y teoriza, tanto en situaciones difíciles como frente a las catástrofes. Pero no es capaz de ponderar adecuadamente las cosas; lejos de observar los defectos o las impurezas de sistemas o teorías, las justifica.

Cacambo mantiene una virtud prudencial con base en su experiencia que parece inspirada en el concepto de la sabiduría popular o el buen sentido común que Aristóteles identificó como la frónesis: actitud práctica y prudente que no se opone a la pretensión de las verdades universales, representadas por la Sofía, sino que más bien se coloca como su conciencia, como el contrapeso necesario de una reflexión razonable -más que sólo racional-y prudente de las pretensiones de universalidad, con base en las particularidades de cada caso. Esta virtud sólo puede adquirirse a través de la experiencia práctica cotidiana.

El Dr. Panglos es una sátira dedicada al optimismo del pensamiento racionalista ilustrado y una burla al sueño cartesiano de lograr un conocimiento claro y distinto a través de la razón. A fin de cuentas, se trata de una crítica al pensamiento moderno, pero especialmente a ese dogma llamado cientificismo.

En este trabajo se pretende mostrar la importancia de la epistemología o de la reflexión epistemológica en todas las disciplinas y en segundo término, realizar una crítica del cientificismo usado de manera acrítica en las ciencias sociales e incluso en las humanidades.

\section{Un mundo ordenado}

Puede sonar trivial la afirmación de que el mundo es maravilloso, como si nuestra capacidad de asombro estuviera ya saturada, producto del exceso de información y de la enorme cantidad de estímulos a los que estamos ex- 
puestos. Aunque también es posible que este desinterés sea consecuencia del sentimiento de incapacidad que nos causa la incomprensión de lo que ocurre a nuestro alrededor. La cotidianeidad está envuelta en prodigios, y no sólo me refiero a los fenómenos naturales, las noches estrelladas, el movimiento del sol, la lluvia o la vida, sino a la tecnología, los autos, los viajes espaciales y las clonaciones.

Se dice que la tecnología la hemos inventado, no existía, y en cambio, a los fenómenos naturales los hemos descubierto; en el fondo de ambas parece haber "mecanismos" que sin fallo alguno hacen que todo funcione. Esta afirmación de que detrás de todo hay una causa se encuentra tan arraigada en nuestra cultura que nos parece imposible creer que algo "funcione" sin causa alguna, es decir, que ocurra un fenómeno sin que exista una razón, una explicación o un fundamento, un mecanismo comprensible, una ley o principio detrás de él, que le dé vida, que lo haga posible. Incluso en la historia, en los acontecimientos que a fin de cuentas son producidos por individuos, observamos tendencias, fuerzas ocultas, una dirección, un destino independiente de los sujetos, al que irremediablemente nos dirigimos. En la economía, sabemos que ese caos de dinero y transacciones también es gobernado por un cerebro oculto: el mercado. Conocemos ya el genoma humano y con ello nuestras fortalezas y debilidades, nuestro destino biológico gobernado por las leyes de la genética.

Algunos afirman que hemos descubierto los derechos fundamentales que son universales e inmutables y por ello son imprescindibles y pertenecen a todos los humanos con independencia de la raza, el credo religioso o la ideología política; hemos descubierto leyes y normas jurídicas y hemos inventado ideologías.

Todo lo natural y lo social está tan ordenado que este fue uno de los argumentos más importantes que emplearon por los filósofos naturales del siglo XVII a favor de la existencia de Dios. De hecho, el estudio de la naturaleza era considerado como una forma de honrar a Dios: si el Creador hizo el mundo ordenado y nos hizo con la capacidad de entender esas regularidades, fue con la intención de que a través de su estudio le conociéramos (Jara, 2009). 
Ahora les invito a repasar un poco las ideas que subyacen en el fondo de los párrafos anteriores. Seguramente muchos se percataron de la fragilidad de mis afirmaciones. Por ejemplo, me referí al movimiento del sol, aunque todos sabemos que el sol no se mueve, sino la Tierra. También mencioné de manera muy superficial que hay cosas que creemos, otras que, dije, conocemos, mencioné cuestiones que descubrimos y otras que inventamos. Hablé de tendencias y leyes, de destinos y de fuerzas ocultas.

Por lo pronto, no es mi intención discutir si lo que afirmé que se descubre es en realidad una invención o si es lo mismo una tendencia que una ley. Mi propósito en este momento es mucho más simple, la de hacer una invitación a reflexionar acerca de las diversas formas que toman nuestras opiniones. A veces son sólo creencias y en ocasiones se trata de creencias justificadas en diversos grados y con diferentes criterios. En ocasiones, incluso, esas creencias parecen ser sinónimo de verdad.

En general, para poner un poco de orden en la terminología, podemos decir que lo que llamamos conocimiento es una creencia justificada. Pero esto no resuelve el problema, porque aún queda pendiente el problema epistemológico fundamental de lo que entendemos por justificación.

\section{El cientificismo}

En general, las discusiones que se dan en el plano estrictamente especulativo y sin referencia alguna a cuestiones concretas pueden ser muy ricas como ejercicios intelectuales pero corremos el riesgo de perdernos en las nubes. En el otro extremo, si sólo discutimos ejemplos de manera casuística nos enredamos en una diversidad caótica que no estoy seguro que sea una mejor meta que las nubes.

Permítaseme recordar en primer lugar los cuestionamientos objeto de la epistemología, y en segundo lugar intentaré ilustrar las razones de las que surgen los temas que se discuten con esa etiqueta, la de la epistemología, y con ello su importancia para todas las disciplinas. Como apunta Dancy (1993, pp. 71-132), las preguntas que se intentan responder en la epistemología son: ¿qué creencias 
están justificadas?, ¿si hay algo que podemos conocer, qué es?, ¿cuál es la relación entre very conocer?, ¿cuál es la relación entre conocimiento y verdad? De modo general podemos resumir la pregunta epistemológica básica a ¿cómo sabemos que sabemos? O bien, ¿cómo justificamos lo que creemos saber?

En todas las culturas se han reconocido una diversidad de formas de obtener conocimiento, es decir, se ha reconocido que existen diferentes tipos de saber, por así decirlo, pero mientras ninguno de ellos mostró superioridad, todos coexistieron de alguna manera, aunque siempre hubo algunos privilegiados por los grupos de poder que lograron predominio relativo. Sin embargo, con la llegada de la ciencia o del conocimiento científico se instauró una forma de conocimiento privilegiada y admirada por sus enormes éxitos, en realidad constituyó una exitosa forma de predicción, de conocer el futuro, pero sobre todo mostró que debajo de las apariencias de un mundo complicado existe un orden extraordinario que, como ya mencioné, muchos interpretaron incluso como la mejor muestra de la existencia de Dios. En dos siglos los logros de la ciencia maravillaron a todos y elevaron la estima del ser humano, que se había percibido a sí mismo como limitado e ignorante. Con la naciente ciencia como ejemplo todos se preguntaron si sería posible imitar el método científico en todas las disciplinas para lograr conquistas semejantes.

Para evaluar adecuadamente lo anterior y no derivar en una discusión en abstracto permítaseme ilustrar con algunos breves ejemplos las sorprendentes conquistas científicas.

Preguntemos: ¿A quién se le pudo ocurrir que la Tierra se mueve? ¿No les parece una locura? ¿Quién siente que se mueve? No hay vientos permanentes en una sola dirección que nos indiquen algún movimiento del planeta, tampoco observamos que al soltar un objeto éste caiga hacia atrás, en la dirección opuesta al movimiento de la Tierra, y nos dicen que la velocidad del planeta es de ¡más de 30 kilómetros por segundo! Todos vemos que el sol sale por el oriente y se oculta por el poniente y observamos también todas las noches el movimiento de las estrellas. ¿Tenía Copérnico alguna evidencia de que la Tierra tuviera movimiento? No. Y si hiciéramos caso a la observación y a la experimentación, creo que la conclusión sería que la Tierra no se mueve. ¿Sabemos que la Tierra se mueve o sólo lo creemos? 
Lo que sentimos es que estamos en reposo, no nos movemos. ¿Cuáles fueron entonces las motivaciones que promovieron la idea de que la tierra se mueve? Es en verdad sorprendente que a alguien se le ocurriera que el planeta se encuentra en movimiento. Lo que se pensaba de manera más o menos generalizada era que el cielo, la esfera celeste, se movía girando a nuestro alrededor, lo que corresponde a lo que observamos todos los días y todas las noches. Sin embargo, los planetas tienen un movimiento raro, avanzan y retroceden; cada uno parece tener un movimiento diferente y particular, y el sol y las estrellas tienen un irregularidad, por decirlo así, no todas las noches del año salen por el mismo sitio: en invierno se "acuestan" hacia el sur. Aunque esto último se puede explicar asumiendo que la esfera celeste realiza una especie de cabeceo durante el año.

Pero para Copérnico y quizá otros, con una mente entrenada en las matemáticas y con la idea firme de que el universo tiene un orden, fue posible la idea de que esas dificultades podían explicarse considerando que la Tierra rota y gira alrededor del Sol.

¿Qué es más atrevido: aceptar que la Tierra se mueve, si no sentimos nada, o aceptar que se mueve una esfera a nuestro alrededor? En realidad, la contienda se da entre la idea de que el mundo está ordenado matemáticamente o no.

La hipótesis de que la Tierra se mueve se vio reforzada con otra idea igualmente excéntrica: no sentimos el movimiento de la Tierra porque simplemente no se siente. $O$ sea, cualquier observación o experimento que se realice en reposo resultará idéntico si se realiza en movimiento constante, es decir, parejito, sin variaciones de velocidad. Lo que quiere decir esto es que lo que observamos en la tierra se nos muestra como si la tierra no se moviera porque se mueve con velocidad uniforme. $Y$ nuevamente el razonamiento de este segundo argumento tiene bases más teóricas que experimentales y fue desarrollado parcialmente por Galileo casi cien años después y posteriormente por Newton en sus Principia, publicados en 1687.

Tiempo después, la primera demostración experimental del movimiento terrestre fue presentada por León Foucault, en 1851, más de trescientos años después de Copérnico. Dicho sea de paso, lo mismo ocurrió con la teoría de 
la relatividad general de Einstein, cuya confirmación experimental se dio varios años después de publicada y aceptada por muchos.

El conocimiento del movimiento terrestre pasó de ser una opinión a una creencia justificada, apoyada en otras creencias, es decir, su justificación no consistió en una corroboración de la teoría con la realidad sino en la coherencia que esta teoría mantenía con otra creencias, especialmente la de que el mundo está ordenado matemáticamente. Pero posteriormente se vio reforzada, no precisamente comprobada -porque se trata de una prueba indirecta- con el experimento del péndulo de Foucault.

El segundo ejemplo de los espectaculares triunfos de la ciencia es el de la caída libre de los cuerpos, también de autoría del señor Galileo. Si hoy día con nuestra tecnología realizamos el experimento de Galileo, los resultados no coinciden con los que él reportó. Galileo afirma que hizo rodar cilindros en un plano inclinado y tomó el tiempo con "pulsaciones"y un reloj de agua. Sí, pulsaciones sanguíneas tocando el cuello o la muñeca, y el reloj de agua consistía en un recipiente con agua colocado en alto con un agujero por el que ésta gotea y es recibida en un recipiente en la parte inferior. El agujero es obstruido con un dedo y se libera al comenzar a medir el tiempo, obstruyendo nuevamente la salida de agua al término de la medición. La cantidad de agua recibida es proporcional al tiempo transcurrido.

Los resultados presentados por Galileo reflejan un orden extraordinario tal, que puede describirse de manera matemática la caída de un objeto y predecirse con enorme precisión el tiempo que tardará en caer. Este resultado es válido si no hubiera aire, pero Galileo lo realizó con aire, por supuesto, pero asumiendo nuevamente un orden intrínseco en la naturaleza.

Pero son las leyes de Newton las que dieron a la Física el prestigio que produjo la envidia de todas las disciplinas y sirvió de inspiración a la llustración. Con solo tres leyes, Newton logró explicar casi todos los fenómenos mecánicos de la naturaleza. Esas leyes son tan poderosas que actualmente se siguen enseñando y siguen siendo válidas para el cálculo de infinidad de problemas físicos. 
Estas leyes que explican adecuadamente los fenómenos, pero que no surgieron tanto de la observación ni de la experimentación, sino sobre todo del uso de la razón, dando por hecho que el mundo y sus fenómenos están ordenados y obedecen a regularidades matemáticas, y que es posible conocer el mundo a través de la búsqueda, haciendo uso de la razón, de principios de los que deriven de manera necesaria los fenómenos observables, su pasado y su futuro.

El tercer ejemplo que deseo comentar contrasta con los anteriores. Se trata de los estudios experimentales que Robert Boyle realizó con el aire y que hoy día se conocen como la ley de Boyle. Lo que Boyle encontró experimentalmente fue una relación muy precisa entre el volumen del aire y la presión que ejerce, sin embargo, nunca admitió que se tratara de una ley matemática porque pensaba que el mundo y sus fenómenos no podían ser tan simples como para ser descritos e incluso predichos a través de las matemáticas. Hoy día, cualquier estudiante de preparatoria que realice el mismo experimento no dudaría en establecer la ley matemática, pero Boyle consideraba que las explicaciones demasiado fáciles y generales de cuestiones tan complicadas sólo oscurecen la inteligibilidad de los fenómenos que, sin embargo, está seguro, pueden ser comprensibles. Sin embargo, también afirmaba que un fenómeno no tiene una sola causa sino causas tan diversas que sólo podemos afirmar con seguridad que son probables (Jara, 2009, pp. 79-121). Por esta actitud prudencial, Robert Boyle fue calificado después de su muerte como alguien poco capaz de develar los verdaderos misterios de la naturaleza (Jara, p. 184), en cambio, Copérnico, Galileo y Newton han compartido el título de padres de la ciencia moderna.

Los dos primeros ejemplos nos muestran el triunfo cartesiano, la aspiración de lograr un conocimiento claro y distinto a través de la razón, deseo de un conocimiento sintético a priori que tanto fascinara a Kant, un tipo de conocimiento que es necesariamente verdadero y universal, incluso antes de ir al mundo de la experiencia, ya que no depende de la experiencia. Su fundamento son las condiciones trascendentales puras, que no sólo no dependen de la experiencia sino que son anteriores a ella y hacen posible su objetividad. 
En cambio, la visión boyleana de una ciencia prudencial fue derrotada, por lo menos hasta muy entrado ya el siglo XX. Pero es interesante hacer notar dos cosas. La primera es que, en esa visión prudencial, la violencia que se vivía en Europa fue un factor muy importante. De hecho, la búsqueda del método científico era vista como la alternativa para dirimir civilmente los conflictos y como un reconocimiento a la falibilidad humana (Stewart, 1992, p. 8), a la incertidumbre inevitable de casi todo el conocimiento humano (Daston, 1988). La segunda cuestión que quiero destacar es el papel del concepto de certidumbre moral, procedente del derecho, que influyó de manera decisiva en lo que hoy denominamos el método científico. La certidumbre moral en los juicios consistía en considerar que cada hecho, cada porción de evidencia o cada testimonio contribuían concurrentemente a la certidumbre, pero sin alcanzar nunca la certeza absoluta.

Sin embargo, desde fines del siglo XVII, el éxito newtoniano y la fascinación por prever el futuro o por conocer nuestro destino promovieron el modelo de ciencia física de las leyes generales o metafísicas, sinónimo de verdad. Había que encontrar regularidades y orden en todo lugar, había que expresarlo con precisión matemática y utilizarlo para predecir exitosamente; desde entonces, el adjetivo de científico se convirtió en una ilusión para todas las disciplinas; todas quisieron ser ciencias, no serlo significaba un status de segunda clase. Esta postura de generalizar el método de las ciencias naturales y especialmente de la Física y aplicarlo en todas las disciplinas como condición para otorgarles el adjetivo de científicas es lo que se conoce como cientificismo.

Beuchot (1993-1994) ha descrito este giro radical entre la Edad Media y la Edad Moderna como el paso de la hermenéutica a la pragmática, como el cambio de una visión mágica, de un saber donde la subjetividad no sólo se interpone entre el intérprete y el libro de la naturaleza, sino que se convierte en un muro infranqueable que oculta la realidad; hacia un nuevo tipo de saber pragmático, racional, que considera posible rescatar el significado del hablante (p. 297), de la naturaleza, que cree en la objetividad de esa interpretación a tal grado que considera posible no tomar en cuenta a la subjetividad. 


\section{Elpositivismo}

El positivismo - término que se usa con una variedad de acepciones, incluso contradictorias, pero que no intento discernir en este momento y que, como afirma Moulines (1979), no consiste en un conjunto de tesis establecidas por escrito en algún sitio, sino más bien en una determinada actitud que ha evolucionado mucho en el tiempo- planteó el rechazo de cualquier pregunta por la esencia de las causas físicas y limitó a la ciencia al establecimiento de relaciones lógico-matemáticas entre los fenómenos; es decir, rechazó toda explicación teológica, metafísica o teleológica de los fenómenos y puso la fe en el progreso acumulativo y continuo de la comprensión científica del mundo como la única forma válida de conocimiento.

Fue un tremendo paso de un extremo a otro. Mientras que en el extremo de la hermenéutica se puede decir que existe siempre un predominio del lector o intérprete, de lo subjetivo, y que entonces existen tantas interpretaciones como intérpretes distintos haya, en la pragmática predomina el autor, su intencionalidad única, la objetividad del mundo y sus leyes, y es, por tanto, unívoco. En la hermenéutica, el sentido, lo que se entiende del texto del mundo, es polisémico; mientras que en la pragmática, la referencia o mundo creado por este texto es unívoca (Beuchot, 1994, p. 149).

Hay que destacar, en este sentido, que todo lo anterior tuvo su base en la consideración de la absoluta madurez y ejemplaridad de las ciencias naturales, sobre todo de la Física y la Química, convicción que se vio mermada con la aparición de la mecánica cuántica y las teorías de la relatividad; con ello, las formas de pensamiento positivista, fundamentalmente centradas alrededor del Círculo de Viena, en los años 1920 a 1930, entrarían en una nueva fase en la que se consideró que el verdadero objetivo de las ciencias no era buscar las causas ocultas de los fenómenos, sino sólo describirlos sistemáticamente, para poder hacer buenas predicciones, con las que la problemática acerca del estatus ontológico y epistemológico de las teorías científicas se acentuó y la infalibilidad del método científico se puso en duda. 
Si ya el movimiento terrestre y las leyes de Newton habían representado una dolorosa ruptura con el sentido común, las nuevas teorías de la relatividad y de la mecánica cuántica aparecían aún más fantasiosas y, sin embargo, increíblemente exitosas. Algunas de las preguntas clave que surgieron entonces fueron: ¿cuál es la relación entre conocimiento científico y verdad o realidad? ¿Son las teorías científicas un fiel reflejo de la realidad o son sólo modelos que permiten predecir exitosamente el futuro? ¿Qué es y cómo se obtiene, descubre, inventa o construye una teoría científica? ¿La observación y el experimento garantizan el conocimiento? ¿Cuál es la relación entre un término teórico y uno observable?

Posiblemente, los tres filósofos de la ciencia más importantes que pusieron en duda la identificación del conocimiento científico con la verdad, después del positivismo, fueron Kuhn, Popper y Feyerabend, a quienes sería necesario dedicar un apartado completo para analizar su pensamiento. Baste por ahora mencionar que sus ideas incorporan elementos que restan objetividad a la ciencia y la colocan como una actividad humana, con frecuencia cercana a la creatividad del arte. Estos filósofos han subrayado, por una parte, que los conocimientos científicos están sustentados invariablemente en paradigmas o supuestos (Kuhn incluso los denomina dogmas) que nunca se ponen en duda, pero que no tienen por qué ser verdaderos. Por otra parte, han destacado la imposibilidad estricta de la verificación o demostración absoluta de un conocimiento a favor de su refutabilidad y finalmente han puesto el énfasis en la construcción social del conocimiento. Feyerabend ha ido aún más lejos, al afirmar que no existe el método científico y defender la anarquía en materia metodológica.

\section{Del caos medieval al caos actual}

Si bien una de las pretensiones de la modernidad, a través de la ciencia, fue la de abandonar los prejuicios medievales, también es cierto que esto permitió el nacimiento de otros ídolos, la ilusión de una objetividad radical, la seguridad de una interpretación única de la realidad y la confianza optimista de que en todos los casos es posible encontrar o des-cubrir la interpretación verdadera, la respuesta correcta. 
Dos supuestos permitieron este movimiento del pensamiento medieval a la racionalidad científica, especialmente en la Física. En primer lugar, la convicción de que la naturaleza no cambia de manera fundamental, que los fenómenos y los objetos naturales son invariantes en esencia, lo que cambia es accidental y poco importante. Esta permanencia esencial de los fenómenos permite el estudio de éstos de manera reproducible y controlada $y$, por tanto, pueden estudiarse como sistemas cerrados o aislados de perturbaciones o efectos externos. De esta forma, en cada fenómeno las variables importantes que intervienen son pocas y conocidas, por lo que pueden ser manipuladas, mientras que se supone que el resto tienen poca influencia y son sencillamente ignoradas.

El segundo supuesto es el de la doctrina de la causalidad (D' Abro, 1951, pp. 45-46). En ésta se da por hecho que la misma situación produce siempre las mismas consecuencias, o dicho de otra manera, que la misma causa produce siempre y exactamente los mismos efectos. En muchos casos esto es cierto, en los fenómenos muy simples, pero en la mayoría de nuestras experiencias, si pudiéramos viajar al pasado y vivir nuevamente, lo más seguro es que, como en la película Corre Lola corre, cualquier pequeñísima modificación produciría cambios enormes. Así que este supuesto aplica solamente a los fenómenos que son absolutamente reproducibles, aquellos que son posibles de reproducir con absoluta precisión a partir de las condiciones en que se originan, denominados condiciones iniciales. Para ello, es necesario reproducir exactamente el inicio del proceso, controlar las variables que le afectan y evitar interferencias externas.

Las dos suposiciones anteriores aplican para fenómenos físicos relativamente sencillos, ordenados y regulares que pueden ser descritos a través de leyes matemáticas; leyes que no sólo los explican sino que además permiten reconstruir su pasado y predecir con singular precisión su futuro. Lo extraordinario de una ley física es que determina a priori la evolución o el desarrollo de un sistema, permite que conozcamos su futuro, y basta para ello conocer con precisión el estado inicial de cosas.

Pero hay que enfatizar que en los sistemas cerrados ideales un pequeño cambio o error en las condiciones iniciales produce un error igualmente pe- 
queño en las predicciones o efectos futuros. Con el tiempo, la realidad nos ha mostrado que el mundo es mucho más complejo, que las dos suposiciones descritas se cumplen en condiciones muy especiales, que los sistemas cerrados son más bien escasos, que la mayoría de los fenómenos se desarrollan en sistemas abiertos y que un pequeño cambio en las condiciones iniciales puede producir efectos insospechados.

El estudio de los fenómenos en sistemas cerrados, aunque supone una simplicidad excesiva, fue lo que permitió a la ciencia mantener válidamente los principios de causalidad, predictibilidad y universalidad, pero a la vez que esos mismos principios posibilitaron un avance sin precedentes en el sabery fueron condiciones para su éxito, pero a la vez que constituyeron los límites inmanentes de la actividad científica moderna, al convertir la metodología científica y sus productos en una especie de dogma, y por tanto, en un obstáculo para el cambio (Kuhn, 1982).

Desgraciadamente, en la realidad no existen los sistemas cerrados. Lo que las ciencias naturales han hecho es construir modelos simples que pueden ser estudiados y que permiten predecir razonablemente los fenómenos bajo estudio. Cuando ha sido posible aislar suficientemente un fenómeno y controlar adecuadamente sus variables ha sido también posible estudiarlo como si prácticamente no interactuara con su exterior, es decir, estudiarlo como un sistema cerrado idealizado. Quizá sólo el universo entero, considerado como un todo, sea el único sistema verdaderamente cerrado.

Aun en la Física y otras ciencias naturales, cuando el estudio de los fenómenos pasó a detalles más finos, la presencia de variables del entorno resultaron ser muy importantes. Pero el descubrimiento más interesante fue que, aun cuando se conozcan las variables y se pueda tener un control más o menos fino de ellas, en algunos sistemas (más de los que se hubiera pensado y deseado) un pequeño cambio produce cambios enormes en los efectos, lo que obligó a abandonar la suposición de que en los sistemas deterministas un pequeño error en las condiciones iniciales se refleja en una pequeña variación en los resultados. 
Estos sistemas abiertos, denominados caóticos, muestran aparente azar, accidentalidad absoluta o comportamiento impredecible, a pesar de estar gobernados por leyes deterministas. Uno de los mejores ejemplos de estos sistemas es el clima: las leyes que lo rigen son conocidas, sin embargo, su predicción es imposible más allá de algunos días. La gran sorpresa fue que estos sistemas pueden ser relativamente simples y que, de hecho, muchos de los fenómenos conocidos pueden mostrar características caóticas.

Desde el descubrimiento de estos sistemas y de su abundancia, nuestra visión del mundo ha cambiado, está cambiando, los sistemas simples pueden dar lugar a comportamientos complejos y los sistemas complejos pueden dar lugar a comportamientos simples (Gleick, 1987, p. 304).

En los denominados sistemas complejos o caóticos, aunque las leyes que los rigen son sencillas y conocidas, tienen un número muy grande de componentes de naturaleza muy distinta; en sus procesos reina la no linealidad: un parámetro puede estar afectado por múltiples causas. En los sistemas complejos, una misma causa puede provocar multitud de efectos (Cereijido, 1996, p. 6).

La suposición de que la regularidad y lo causal determinista eran la norma y lo contingente de un accidente llevó a considerar el mundo como si se tratara de un sistema lineal, que podía estudiarse en pedazos. Se estudia independientemente cada trozo y luego simplemente se juntan. Pero en los sistemas no lineales la suma de las propiedades de cada parte no es igual a las propiedades del todo.

Las interacciones entre las partes de un sistema no lineal dan lugar a propiedades emergentes, nuevas, que no pueden explicarse a partir de las propiedades de las componentes. Un ejemplo es un hormiguero, donde la colonia de hormigas es capaz de desarrollar tareas sociales, es decir, tareas que sólo son posibles de desarrollar en comunidad, pero que cada hormiga por sí sola no realizaría en parte proporcional. El comportamiento social emergente es característico de un sistema complejo y no se puede reducir a las propiedades de cada individuo. 
El premio Nobel de Química, llya Prigogine, ha hecho énfasis en que en esos casos, con frecuencia, aparecen elementos azarosos, como si no hubiera leyes, lo que muestra que incluso la realidad física es mucho más que la suma de las partes estudiadas por separado, que existe una diferencia cualitativa entre las partes y el todo. Reconocer esa complejidad y efectuar una relectura dentro de este nuevo contexto de relaciones cambiantes del hombre con la naturaleza y de ésta con el hombre es, dice Prigogine (1983), uno de los problemas cruciales de nuestra época (pp. 46-54).

Las ciencias sociales no estuvieron exentas de la influencia cientificista y adoptaron modelos semejantes a los de las ciencias naturales. Pero mientras que algunos fenómenos físicos se pueden reproducir fielmente y entonces las teorías pueden verificarse o falsarse experimentalmente, en la Historia, el Derecho y otras disciplinas, cada evento es único e imposible de repetir, por lo que pretender utilizar la metodología de la Física conlleva un reduccionismo extremo y la negación de la complejidad intrínseca de los fenómenos sociales en los que el conocimiento que podemos obtener sobre toda nuestra experiencia pasada es, como bien ha dicho Roy (1997), similar a asomarse de noche a una casa vieja por la ventana: vemos cuadros sobre la pared y libros, nuestros ancestros están dentro, pero no podemos entrar y lo que observamos son sólo sombras y lo que escuchamos sólo son susurros.

\section{E}

Pues bien, pasemos ahora a analizar la relevancia del papel de la epistemología; a pesar de todo, siguen siendo válidas y relevantes las preguntas de cómo es que eso que denominamos ciencia funciona, cómo es posible obtener conocimiento seguro o lo más seguro posible acerca del mundo; y por otro lado, de qué manera justificamos nuestras creencias o de qué manera se justifican las pretensiones de conocimiento que nos enseñan desde la escuela hasta los medios de comunicación. Sería un grave error echar por la borda la experiencia humana y afirmar sin más que todo el saber es relativo y subjetivo e identificar cualquier opinión (que pudiera ser religiosa, política o simplemente charlatanería) con el conocimiento; a fin de cuentas, debe existir alguna diferencia que pudiera ser relevante, incluso para la supervivencia humana. 
La invitación es a pensar críticamente, de manera creativa y constructiva, sin descalificar nada a priori ni de manera simplista, abriendo en lo posible nuestra comprensión para lograr entender los posibles propósitos de nuestros interlocutores, ya sean los textos, nuestros colegas o nuestros estudiantes. Como ha dicho Feyerabend (1999), cualquier idea tiene fallos e incluso la idea más tonta tiene aspectos interesantes (p. 86), y si en este caso estamos ante ideas discutidas por personajes intelectualmente notables seguramente podremos aprovecharlas.

La justificación de una creencia o las razones por las que podemos afirmar que un conocimiento es verdadero o que es cuando menos razonable, plausible o lo suficientemente verosímil para mantener la confianza entre la racionalidad y el conocimiento, es lo que pretenden explicar las diversas posturas epistemológicas, en la búsqueda de una garantía que nos permita distinguir una mera opinión de un conocimiento. De lo que se trata entonces es de explicitar las razones de por qué consideramos una creencia más racional o más fiable que otra.

Las dos posiciones epistemológicas clásicas son el fundamentalismo y el coherentismo en la estructura de la justificación de una creencia. Mientras que el fundamentalismo afirma que existen creencias básicas que no requieren justificación alguna porque son auto evidentes y sirven de base o cimiento a otras, los coherentistas consideran que lo que hace válida o justifica una creencia es su relación, armonía o consistencia con otras creencias (Dancy, 1993, pp. 71-132). Por supuesto que estas no son las únicas posturas pero son, de alguna manera, las más básicas. A estas debe agregarse que la hermenéutica considera que, si bien el objetivo del conocimiento de los fenómenos naturales es su explicación con fines predictivos, en el conocimiento social el objetivo es distinto, no se trata de explicar sino de comprender, porque son fenómenos mucho más complejos que los naturales. Para Gadamer (1991), por ejemplo, la experiencia del comprender es irreductible al método científico que busca un saber exacto y objetivo. Probablemente haya eventos sociales que pueden presentar regularidades o tendencias y un orden tal que sea dable tratarlos como si se tratara de un fenómeno natural, pero no en todos los casos esto es posible y, en general, en un fenómeno social las 
tendencias o regularidades corresponden sólo a una parte o componente del fenómeno.

Regresando a los breves ejemplos que mostramos, lo que se observa es que, por lo menos en el caso de las teorías físicas, que han resultado suficientemente apegadas a la verdad, su justificación ha sido, en principio, más bien coherentista aunque se hayan fundamentado empíricamente a posteriori. Incluso la ley de Boyle, que sin duda se trata de un conocimiento justificado en la experimentación, no deriva directamente de la pura experiencia, prueba de ello es que el mismo Robert Boyle no "observó" ninguna ley matemática, sino solamente una regularidad particular. Para "observar"la "Ley" se requiere de una interpretación adicional que acepte que el mundo natural está ordenado y obedece, por así decirlo, a una realidad matemática.

\section{Consideraciones finales}

Aunque la ciencia moderna parecería moverse con una ilusión pragmática en su tarea de interpretar el libro de la naturaleza, tiene la pretensión y la confianza de lograr una objetividad tal que rescate la intencionalidad de las leyes naturales; sin embargo, cuando en su pretensión reconoce que no logrará apresar de manera completamente objetiva las leyes naturales porque se interpone irremediablemente la subjetividad del científico como intérprete, entonces su trabajo se hace hermenéutico (Beuchot, 1993-1994, p. 295), un trabajo de interpretación no unívoco. Así, el fenómeno de la comprensión rebasa el ámbito filosófico y aparece con validez dentro de las ciencias naturales. En realidad, el problema se remite a la discusión ya clásica acerca de los términos teóricos, de la neutralidad de la observación y de la problematicidad de las teorías.

Lo unívoco es lo que no tiene vuelta de hoja, se entiende por sí mismo, no requiere interpretación porque su sentido es único, objetivo, comprendido por todos y cada uno. Lo unívoco es un libro en el que la intencionalidad del texto, la del autor y la del lector se corresponden; se trata de lo mismo. La afirmación de la univocidad en las leyes de la naturaleza es maravillosamente expresada por Lagrange, al reconocer el genio de Newton por haber sido 
quien interpretara las leyes del universo. Decía Lagrange:"Hay solamente un universo, puede suceder que sólo un hombre en la historia del mundo sea el intérprete de sus leyes" (citado por Kuhn, 1979, p.13).

La objetividad de la ciencia, como garantía o sinónimo de su univocidad, da lugar a la concepción de que se trata del saber más valioso. El cientificismo constituye la posición radical de univocidad. Lo que se trata de criticar entonces es la reducción de la verdad al ámbito del método científico (Vattimo, 1991, p.68), que invalida cualquier otro tipo de saber y se puede convertir en una limitación para el propio avance de la ciencia.

En la producción del discurso científico es conveniente buscar coherencias y regularidades que eviten la equivocidad, hacer de ellas el objeto científico y con ello reducir la carga interpretativa. Pero dado que es imposible obtener los conocimientos puramente objetivos sino que necesariamente aparecen en los fenómenos pequeñas irregularidades, excepciones a la ley, a la norma, a lo unívoco, la ciencia debe tomar esas anomalías como meros accidentes, como defectos superables; no niega esas anomalías, pero las muestra como un efecto de superficie, de tal manera que la contradicción aparezca como sólo una ilusión o una característica secundaria y deje el primer sitio a las equivalencias y a las similitudes. Las coherencias juegan un papel tan fundamental en la producción científica que la ciencia echa mano de toda su creatividad para agruparlas, encontrarlas o construirlas de las formas más heterogéneas y diversas, dando lugar a leyes o regularidades de naturaleza muy diferente (Foucault, 1991, pp. 251-252). De esta manera, aunque la ciencia pretende estar en la univocidad, se encuentra en un terreno intermedio entre la equivocidad y la univocidad.

Para concluir quisiera hacer dos comentarios y dos advertencias. El primer comentario es que hoy día las regularidades matemáticas simples con las que la Física clásica describe el mundo son vistas sólo como aproximaciones de un comportamiento mucho más complejo. El segundo es que la pretensión de cientifizar todos los saberes en el sentido de explicar los hechos y los procesos de su competencia a través de regularidades generales que permitan formular leyes y predecir el futuro es una simplificación aun mayor que la que la Física realizó hasta antes del siglo XX. 
La primera advertencia es que las ciencias naturales se han modificado radicalmente frente al descubrimiento de un mundo tan complejo; quizá una excelente manera de describir las nuevas ciencias naturales sea en palabras de un físico laureado con el premio Nobel, llya Prigogine, quien afirma que tanto en las ciencias físicas como a fortiori en las ciencias humanas, ya no es admisible la idea de realidad como algo dado (Prigogine, 1983, p. 45), y agrega con Stengers: que la ciencia de hoy no puede ya adjudicarse el derecho de negar la pertinencia y el interés de otros puntos de vista, de negarse en particular a escuchar los de las ciencias humanas, de la filosofía y del arte (Prigogine y Stengers, 1990, p. 84).

La segunda advertencia es que, si bien en las ciencias naturales se ha redescubierto la complejidad del mundo y se reconoce que a fin de cuentas todo conocimiento es un producto humano y no un mero descubrimiento en el sentido de que no des-cubrimos el mundo sino que necesariamente lo interpretamos, lo anterior no demerita el trabajo científico ni nos exime de pensar responsablemente, analizar y justificar lo mejor posible nuestras pretensiones de conocimiento, so pena de caer en la trampa y otorgar el mismo estatus a un conocimiento bien justificado que a la opinión de un charlatán. Permítaseme expresar lo anterior, nuevamente, con palabras prestadas, en esta ocasión las del filósofo Laudan (1996), quien nos advierte del peligro del reemplazo de la idea de que los hechos y la evidencia tienen importancia, por la de que todo se reduce a intereses y perspectivas subjetivos, que es la manifestación más prominente y perniciosa de un peligroso anti-intelectualismo de nuestros tiempos.

\section{Referencias}

Beuchot, M. (1993-1994). Reflexiones sobre la Retórica desde la Hermenéutica. Acta Poética, (14-15).

Beuchot, M. (1994). Naturaleza y Operaciones de la Hermenéutica según Paul Ricoeur. Pensamiento, 50 (196). 
Beuchot, M. et al. (1995) Los márgenes de la interpretación: hacia un modelo analógico de la hermenéutica. Universidad Iberoamericana. Cuadernos de Filosofía, (25).

Cereijido, M. (1996). Del Caos de los Demonios al Caos de los Biólogos. Revista Universidad de México, 1, 3-7.

D'Abro, A. (1951). The rise of the new physics. New York, EE. UU.: Dover Publications.

Dancy, J. (1993). Introducción a la epistemología contemporánea. Madrid, España: Tecnos.

Daston, L. (1988). Classical probability in the enlightment. Princeton, EE. UU.: Princeton University Press.

Feyerabend, P. (1999). Ambigüedad y armonía. Barcelona, España: Paidós.

Foucault, M. (1991). La arqueología del saber, México: Siglo XXI.

Gadamer, H. G. (1991). Verdad y método. Salamanca, España: Sígueme.

Gleick, J. (1987). Chaos: making a new science. New York, EE. UU.: Penguin Books.

Jara, S. (2009). La ciencia prudencial de Robert Boyle. Morelia, Michoacan de Ocampo, México: Universidad Michoacana de San Nicolás de Hidalgo.

Kuhn, T. (1979). La función del dogma en la investigación científica. Valencia, España:Teorema.

Laudan, L. (1996). Beyond positivism and relativism. Theory, method, and evidence. Boulder, Colorado, EE. UU.: Westview Press.

Moulines, C. U. (1975). La Génesis del Positivismo es su Contexto Científico. Dianoia: anuario de filosofía, (21), 31-49.

Prigogine, I. (1983). ¿Tan sólo una ilusión? Barcelona, España: Tusquets Edi- 
tores.

Prigogine, I. y Stengers, I. (1990). La nueva alianza, metamorfosis de la ciencia. Madrid, España: Alianza.

Roy, A. (1997). The god of small things. New York, EE. UU.: Random House.

Stewart, L. (1992). The rise of public science. Cambridge: Cambridge University Press.

Vattimo, G. (1991). Ética de la interpretación. Barcelona, España: Paidós. 